\title{
Research on Aeolian Vibration Fatigue Life of Conductors
}

\author{
$\mathrm{LI} \mathrm{Li}^{1,}$,, ZHANG Zuyan ${ }^{1, b}$, XU Ningbo ${ }^{1, c}$ \\ ${ }^{1}$ School of Civil Engineering \& Mechanics, Huazhong University of Science \& Technology, Wuhan, \\ 430074, China \\ aili2431@163.com, 르201573140@hust.edu.cn, xnb_hust@163.com
}

\begin{abstract}
Keywords: Aeolian vibration, Nominal stress method, The energy balance method, Fracture mechanics method, Stockbridge-type damper.
\end{abstract}

\begin{abstract}
The transmission lines' fatigue failure caused by aeolian vibration is increasingly apparent as the wide applications of ultra-high voltage, so it is necessary to study the conductors' fatigue life of aeolian vibration. Different from the traditional infinite life design method and nominal stress method, the energy balance method is used to calculate the amplitude of the aeolian conductor vibration and fracture mechanics method is used to calculate the cycle times required for the fatigue failure of conductors. Based on the probability distribution theory of wind speed, the number of cycles of the aeolian vibration at a certain frequency is calculated. Then, the fatigue life of aeolian vibration is calculated combined with Miner linear damage theory. Comparing with the result of the nominal stress method, we can find that the nominal stress method is conservative. Finally, the fracture mechanics method is used to calculate the fatigue life of the conductor with Stockbridge-type dampers, which lays the foundation for the anti-vibration analysis of the aeolian vibration of conductors.
\end{abstract}

\section{Introduction}

High-voltage transmission lines are directly related to China's economic construction and national defense construction and play an important role in optimizing the allocation of power resources. It is known as blood vessels of the national power. All along, problems about fatigue failure of conductors caused by the aeolian vibration are more concerned by domestic and foreign researchers. With the increase of transmission lines' span and tension, the aeolian vibration is more likely to happen. As a result, the abrasion of fittings and the conductor breakage caused by fatigue occur frequently [1-3]. Therefore, it is of great practical significance to study the aeolian vibration fatigue life of conductor.

The aeolian vibration of a conductor is also called the vortex-induced vibration. Comparing with the subspan oscillation of multi-bundle conductors and the galloping of iced conductors, frequency of the aeolian vibration is higher and amplitude of that is smaller. The frequency of vibration is generally between 3 and $150 \mathrm{~Hz}$, the maximum amplitude is often no more than 1 to 2 times of the conductor diameter. At present, some scholars at home and abroad have calculated and analyzed the response of the aeolian vibration and the fatigue life of conductor. In order to calculate the response of aeolian vibration of conductor, the energy balance method [4,5] is widely used for the calculation at home and abroad. The basic principle of this method is that the energy input to the conductor is equal to the energy dissipated by conductor-damper coupling system, and the equilibrium amplitude of the conductor is calculated. Combined with the theory of forced vibration analysis, $\mathrm{Lu} \mathrm{[6]} \mathrm{used} \mathrm{impedance} \mathrm{matrix} \mathrm{transformation} \mathrm{method} \mathrm{to} \mathrm{analyze} \mathrm{the} \mathrm{vibration} \mathrm{response} \mathrm{of}$ conductor-damper system and this method has a high accuracy and efficiency. A single threshold method was adopted by Zhongnian $\mathrm{Xu}$ [7] to evaluate the fatigue life of transmission lines. Deyi Kong and Guanghong Zhu [8,9] used the nominal stress method to calculate the aeolian vibration fatigue life of conductor. Haobin Chen [10] used fracture mechanics method to calculate the number of alternating stress cycles which is required for the fatigue failure of, and the calculation results were compared with the experimental results. 
Although the above scholars have done a full study on the aeolian vibration response and fatigue life of the transmission lines, there are still some unsolved problems. The single threshold method is based on infinite life design method and cannot give the value of the aeolian vibration fatigue life of conductor quantitatively. The nominal stress method often replaces the real S-N curve of the material with the wöhler safety curve, making the results too conservative.

In this paper, the calculation method of fatigue crack propagation in fracture mechanics is used to calculate the aeolian vibration fatigue life of conductor. Firstly, basic principles of the energy balance method and the fracture mechanics method are simply stated, and the number of alternating stress cycles required for fatigue damage of conductor is calculated. Secondly, based on the theory of wind speed probability distribution, the corresponding numbers of cycles of the aeolian vibration response are given. Then, combined with Miner's linear damage theory, the vibration fatigue life of the conductor is calculated and compared with that obtained by the nominal stress method. Finally, the method is used to calculate the fatigue life of conductor with dampers touched in the actual engineering, which can lay a foundation for anti-vibration analysis of aeolian conductor vibration.

\section{The Energy Balance Method}

Basically, the aeolian vibration is attributed to the process of vortex shedding on the leeward side of conductor when the wind is passing through the conductor. As a result of the vortex shedding, an alternating lift force in the vertical direction of the power transmission line is produced. When the frequency of the alternating lift force is close to the natural frequency of the conductor, the conductor will resonate. The basic principle of the energy balance method is that when the conductor resonates, the energy input from the wind load to the conductor vibration system is equal to the energy dissipated inside the wire vibration system and the steady-state amplitude of the aeolian conductor vibration is determined.

The wind energy input to the conductor per unit time is denoted by $P_{w}$, which is called wind power. The internal power consumption of the conductor vibration is expressed by $P_{c}$, which is called the self-damping power of the transmission lines. The power dissipated by the vibration-proofing device is represented by $P_{d}, P_{w}, P_{c}$, and $P_{d}$ are both functions of amplitude $A$ and frequency $f$. For a fixed frequency $f_{0}$, they are only a function of amplitude $\mathrm{A}$, as shown in Fig. 1.

Wind Power. The results show that when the aeolian conductor vibration occurs, the wind energy input to the conductor is related to its structural parameters and wind speed. Further studies have shown that the lift force acting on the conductor which is due to vortex shedding varies sinusoidally. The lift force can be expressed as the following equation:

$$
F=F_{y} \sin \omega t=C_{L} \frac{1}{2} \rho V^{2} D \sin \omega t
$$

Where, $F_{y}$ is the amplitude of the lift force; $C_{L}$ is the lift coefficient; $\rho$ is the air density; $V$ is the wind speed; $D$ is the conductor diameter; $\omega$ is the circular frequency of lift force.

The vertical displacement of the aeolian conductor vibration can be defined as $y=-A_{0} \cos (\omega t-\varphi)$. The average value of the energy delivered to the unit length conductor per unit time by the wind is given by the following equation.

$$
P_{w}=\frac{2}{T} \int_{0}^{\frac{\pi}{2}} F d y=\frac{1}{2} F_{y} \omega A_{0} \cos \varphi
$$

Where, $\omega=2 \pi f$. When the conductor resonance occurs, $\cos \varphi=1$. So

$$
P_{w}=\pi F_{y} A_{0} f=\frac{1}{2} C_{L} \rho V^{2} D \pi A_{0} f
$$

At present, the research results about wind input power by Diana \& Falco [11, 12] are the most widely used in the country. The wind input power using the formula (4) shows the expression: 


$$
\begin{cases}P_{W}=e^{\left(a_{1} \ln \left(y_{0} / D\right)^{2}+a_{2} \ln \left(y_{0} / D\right)+a_{3}\right)} f^{3} D^{4} \quad\left(y_{0}<1.2 D\right) \\ P_{W}=74.31 f^{3} D^{4} & \left(1.2 D \leq y_{0}<2.0 D\right) \\ P_{W}=0 & \left(y_{0}>2.0 D\right)\end{cases}
$$

Where $a_{1}=0.0526, a_{2}=1.4074, a_{3}=4.0324$ are constants; $y_{0}$ is double amplitude of the conductor; $D$ is the conductor diameter.

Self-damping Power of Conductor. The power dissipation due to conductor self-damping represents the capacity of consuming or absorbing vibration energy during aeolian vibration process. It is also a major source of energy dissipation during aeolian vibration. The greater the value of the self-damping power, the more advantageous is the anti-vibration. There are many factors affecting the power dissipation due to conductor self-damping, such as aeolian vibration amplitude and frequency, conductor tension, environment temperature and conductor material etc.

At present, the literature on the theoretical calculation of the self-damping power of the conductor is less, and the test is generally taken. In the experiment of measuring self-damping power, domestic scholars usually use the expression as follows:

$$
P_{c}=K f^{\beta}\left(\frac{y_{0}}{D}\right)^{\alpha}
$$

Where, $P_{c}$ is the power dissipation due to conductor self-damping; $f$ is vibration frequency; $y_{0}$ is the double vibration amplitude; $D$ is the conductor diameter; $K, \alpha, \beta$ are the test coefficient related to frequency.

Power Consumption of Dampers. A Stockbridge-type damper transforms vibration energy it absorbs into thermal energy and acoustic energy and they are dissipated, reducing the steady-state amplitude of aeolian vibration of a conductor-damper system. The larger value of power dissipation, the stronger inhibitory effect that a Stockbridge-type damper has on aeolian vibration of conductor is.

Performance of a Stockbridge-type damper is represented by its curve of power characteristics [12]. The power characteristics are generally measured by the experiment. Because of the limited experiment conditions, the theoretical method is adopted to analyze the dynamic characteristics of a Stockbridge-type damper in this paper.

The mathematical model of Damper is shown in Fig. 2. Where, $O$ is the hammer centroid; $O^{\prime}$ is the inside clamping point of hammer and strand; the $L$ is the distance between damper clamping point and the inside clamping point of hammer and strand; $G$ is the distance between hammer centroid and the inside clamping points of hammer and strand; $y(t)$ is the displacement of the inside clamping point of hammer and strand with damper clamp as a reference system; $\varphi(t)$ is the angle of hammer rotation; $u(t)$ is the displacement of damper clamp.

Based on the above assumptions, the equation of motion about dynamic characteristics of the independent subsystem can be expressed as follows:

$$
\boldsymbol{M} \ddot{\boldsymbol{X}}+\boldsymbol{C} \dot{\boldsymbol{X}}+\boldsymbol{K} \boldsymbol{X}=\boldsymbol{F}
$$

Where, $\boldsymbol{M}, \boldsymbol{C}$ and $\boldsymbol{K}$ are mass matrix, damping matrix and stiffness matrix of Damper independent subsystem, $\boldsymbol{X}$ is displacement array; $\boldsymbol{F}$ is external force array.

Here,

$$
\boldsymbol{M}=\left[\begin{array}{ll}
M_{11} & M_{12} \\
M_{21} & M_{22}
\end{array}\right], \quad \boldsymbol{C}=\left[\begin{array}{ll}
C_{11} & C_{12} \\
C_{21} & C_{22}
\end{array}\right], \quad \boldsymbol{K}=\left[\begin{array}{ll}
K_{11} & K_{12} \\
K_{21} & K_{22}
\end{array}\right], \quad \boldsymbol{X}=\left[\begin{array}{c}
y \\
\varphi
\end{array}\right], \quad \boldsymbol{F}=\left[\begin{array}{c}
-m \ddot{u} \\
0
\end{array}\right] .
$$

Where, $M_{11}=m ; M_{12}=-m G ; M_{21}=0 ; M_{22}=J ; K_{11}=12 E I / L^{3} ; K_{12}=-6 E I / L^{2}$;

$K_{21}=-6 E I / L^{2}+12 E I G / L^{3} ; \quad K_{21}=4 E I / L-6 E I G / L^{2} ;$

Here, $m$ is the hammer mass; $J$ is the rotational inertia of hammer; $E I$ is the strand bending stiffness. 
The damping matrix $\boldsymbol{C}$ can be derived from the stiffness matrix $\boldsymbol{K}$ as follows:

$$
\boldsymbol{C}=\frac{2 D}{\omega} \boldsymbol{K}
$$

Where, $D$ is the damping ratio of system; $\omega$ is the circular frequency of vibration.

Considering the steady vibration, $\boldsymbol{X}$ can be solved as follows:

$$
\boldsymbol{X}=\left(-\boldsymbol{M} \omega^{2}+\boldsymbol{K}+j \omega \boldsymbol{C}\right)^{-1} \boldsymbol{F}
$$

Here, $j$ is the imaginary unit.

Displacement of damper clamp vibration $u=u_{0} \sin (\omega t)$, so the solution can be derived as follows:

$$
\left\{\begin{array}{c}
y \\
\varphi
\end{array}\right\}=\left(-\boldsymbol{M} \omega^{2}+\boldsymbol{K}+j \omega \boldsymbol{C}\right)^{-1}\left\{\begin{array}{c}
m \omega^{2} u \\
0
\end{array}\right\}
$$

After $y$ and $\varphi$ solved, the relationship of damper resistance and displacement is $F=K_{11} y+K_{12} \varphi$ and the power consumption of dampers is $P=\frac{1}{2} \omega|F| \cdot|u| \cos a$. MATLAB language is used to write the simulation program of damper power characteristics. Specific flow chart is shown in Fig. 3.

\section{Fracture Mechanics Method}

Fatigue fracture of conductors caused by the aeolian vibration is a kind of fretting fatigue. Due to fretting, Wear and tear on contact surface between the conductor strands lead to the initiation of conductor fatigue crack, and the proportion of fatigue crack initiation life of total life greatly reduced. It can be considered that the total fatigue life of the conductor is the fretting crack development life. In this paper, fracture mechanics method is used to study the crack development life of transmission lines, the basic formula is as follows:

$$
N=\int_{a_{0}}^{a_{c}} \frac{d a}{C(\Delta K)^{n}}
$$

Where, $a_{0}$ is the initial crack length; $a_{c}$ is the critical crack length; $\Delta K$ is the amplitude of stress field intensity factor; $C, n$ are material constants associated with pure aluminum.

After determining the above parameters, the cycle times required for the crack length extending from $a_{0}$ to $a_{c}$. That is the fatigue life of conductor.

In order to get the value of $\Delta K$ in equation (10), it is necessary to simplify the single-stranded conductor into a finite width lath to calculate the length of the crack. Due to the errors caused by the difference between the two shapes, a correction coefficient $\eta$ can be introduced for correction. For the values of $a_{0}$ and $a_{c}, a_{0}$ should be chosen as the smaller value of the depth of fatigue crack in the fretting-mixing zone, and the $a_{c}$ should be chosen as the larger value of the length of fatigue crack in the fatigue crack growth zone. The cycle times required for fretting wear and crack initiation on conductor surface and the cycle times required for the conductor fracturing at final stage are added to the cycle times required for the fretting fatigue crack growth, and the prediction accuracy of fatigue life [10] is improved

According to the literature [10], $a_{0}=20 \mu \mathrm{m}, a_{c}=2000 \mu \mathrm{m}$. $C$ and $n$ are parameters related to properties of pure aluminum, taking $C=3 \times 10^{-7}, n=3.1$. The value of $\Delta K$ can be obtained by the following equation:

$$
\Delta K=F \sigma \sqrt{\pi a}
$$

Where, $a$ is the length of growth crack; $\sigma$ is the tensile stress of members; $F$ is the shape 
modification coefficient of finite width laths. The empirical formula is expressed as follows:

$$
F=1.12-0.231\left(\frac{a^{\prime}}{b}\right)+10.55\left(\frac{a^{\prime}}{b}\right)^{2}-21.72\left(\frac{a^{\prime}}{b}\right)^{3}+30.39\left(\frac{a^{\prime}}{b}\right)^{4}
$$

Where, $a^{\prime}$ is the preset length of crack, taking $a^{\prime}=a_{0}=20 \mu \mathrm{m} ; b$ is the width of finite width laths, taking $b=2000 \mu \mathrm{m}$; Substituting (11), (12) into (10) yields

$$
N=\frac{2}{C(n-2)(F \sqrt{\pi})^{n}(\Delta \sigma)^{n}}\left(a_{0}^{1-\frac{n}{2}}-a_{c}^{1-\frac{n}{2}}\right)
$$

When the shape correction factor $\eta$ and the stress parameter $\delta$ are introduced, the cycle number $N_{f}$ required for the fretting fatigue crack growth is:

$$
N_{f}=\delta \eta N
$$

Setting $\beta=\delta \eta, \beta$ is the comprehensive parameter factor. The cycle number $N_{f}$ can be expressed as the following equation.

$$
N_{f}=\beta N
$$

$N_{f}$ can be obtained by finding the stress amplitude $\Delta \sigma$ and the comprehensive parameter factor $\beta$.

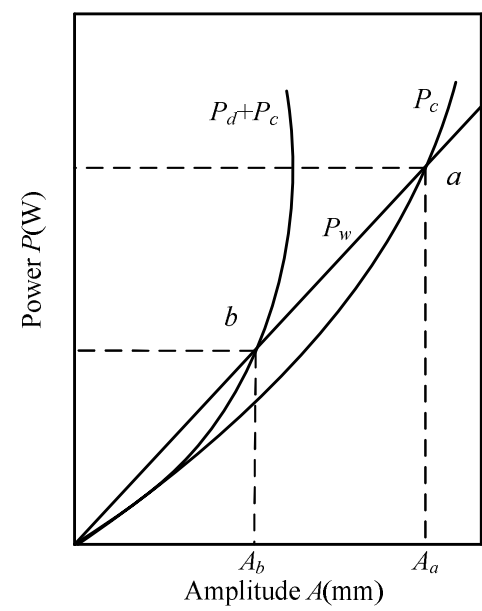

Fig. 1 Diagram of the energy balance method.

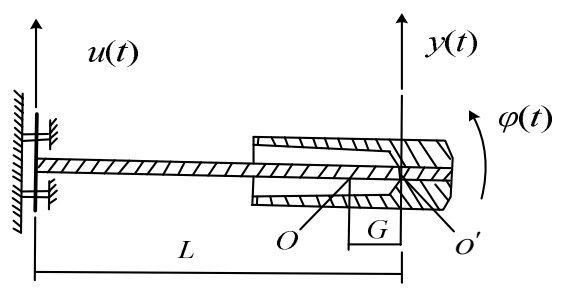

Fig. 2 Theoretic calculation model of a Stockbridge-type damper.

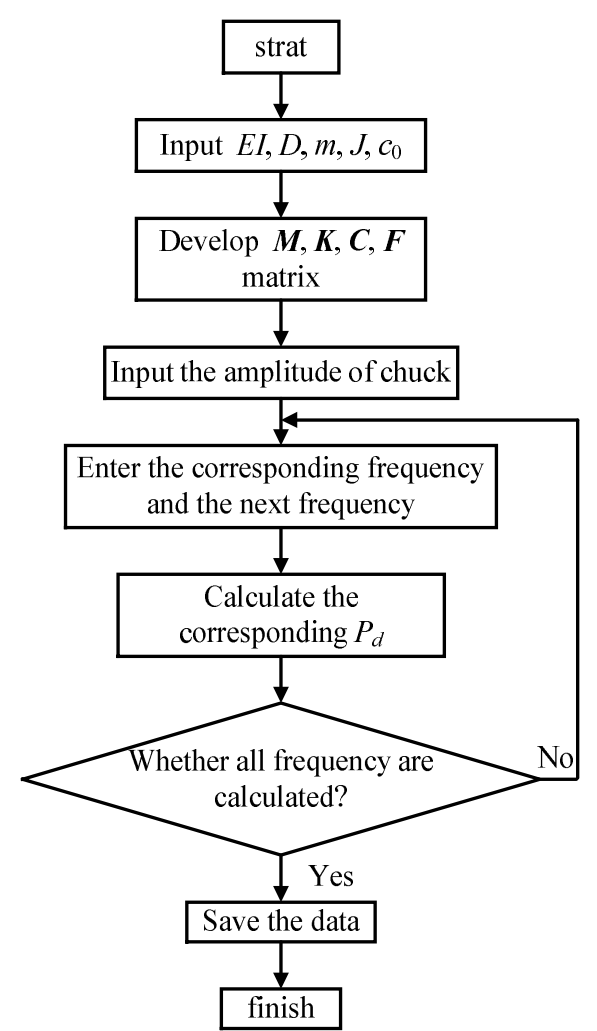

Fig. 3 The flow chart of calculation of characteristics of the damper.

\section{Probability Distribution of Wind Speed}

One of the conditions for the occurrence of aeolian vibration of the conductor is that there is a comparatively stable Carmen vortex shedding frequency. When the wind speed and wind direction change, the frequency of the aeolian conductor vibration will also change, and the different 
frequency corresponds to the different fatigue life of the conductor. Therefore, the variation of wind speed should be taken into consideration when calculating the aeolian vibration fatigue life of conductors.

Wind speed is a random variable, so the frequency of conductor vibration is a function of random variables. In fact, the wind speed at the same time cannot be the same for the whole span of the conductor, but as a result of the "locking effect" of the aeolian conductor vibration, the amplitude of change of wind speed does not exceed $\pm 20 \%$, the vibration frequency of transmission lines can be considered the same.

At present, there are many probability distribution models of estimation of wind speed. The research results of many scholars and a large number of measured data show that Weibull distribution model is very effective in fitting wind speed, so this adopted is used as the probability distribution model of average wind speed. The commonly used two-parameter Weibull distribution function is as follows:

$$
P\left(U \leq U_{c}\right)=1-\exp \left[-\left(U_{c} / c_{w}\right)^{k_{w}}\right]
$$

Where, $P\left(U \leqslant U_{c}\right)$ is the probability which is less than $U_{c} ; k_{w}$ is a shape parameter, which can be calculated by the following equation.

$$
k=0.74+0.19 \bar{v}
$$

Where, $\bar{v}$ is the average wind speed for many years.

When the aeolian vibration of conductor occurs, the relationship between the vibration frequency $f_{c}$ and the wind speed $U_{c}$ is shown in formula (18)

$$
f_{c}=S_{t} U_{c} / D
$$

Where, $S_{t}$ is the Strouhal number, generally taking 0.2; Combining equations (16) and (18), $P(U \leqslant$ $U_{c}$ ) can be given as follows:

$$
P\left(f \leq f_{c}\right)=-\exp \left[-\left(f_{c} D / S_{t} c_{w}\right)^{k_{w}}\right]
$$

The wind speed which can cause the aeolian conductor vibration is generally $0.5 \sim 10 \mathrm{~m} / \mathrm{s}$. The frequency of the vibration is generally between 10 and $100 \mathrm{~Hz}$. The frequency range in which the aeolian vibration occurs is divided into a plurality of small frequency bands, and the probability that the frequency is in the $\mathrm{i}^{\text {th }}$ segment is:

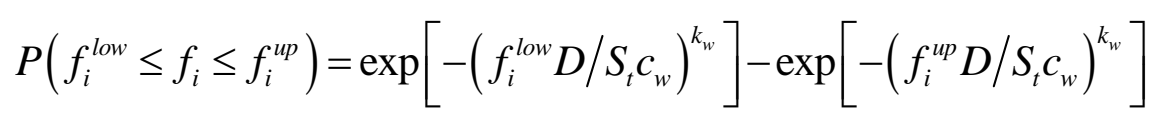

Here, $f_{i}^{u p}$ is the upper limit of the frequency in the $i^{\text {th }}$ segment; $f_{i}^{\text {low }}$ is the the lower limit of the frequency. Within one year, the time in which the vibration frequency is in the $i^{\text {th }}$ segment can be expressed as follows:

$$
t_{i}=3.1536 \times 10^{7} \times\left\{\exp \left[-\left(f_{i}^{\text {low }} D / S_{t} c_{w}\right)^{k_{w}}\right]-\exp \left[-\left(f_{i}^{u p} D / S_{t} c_{w}\right)^{k_{w}}\right]\right\}
$$

Adding up the vibration time in each frequency band, the total vibration time is obtained as follows:

$$
t=\sum t_{i}
$$




\section{Miner Linear Damage Theory}

Failure of most engineering structures results from accumulation of fatigue damage caused by a series of cyclic loads of variable amplitude. The cumulative fatigue damage theory is about studying fatigue damage accumulation rule and failure criterion under the variable amplitude fatigue load. It is very important to predict the fatigue life of structure. In general, the theory of fatigue cumulative damage is divided into linear theory and nonlinear theory. The linear theory is simple and easy to calculate, and it is widely used in metal fatigue life estimation. Therefore, the linear damage theory is chosen to calculate the aeolian vibration fatigue life of conductor in this paper.

The Calculation Principle of Miner Linear Damage Theory. Theory of linear fatigue damage accumulation is based on the following two calculation principles:

(1) Under the action of cyclic load, the fatigue damage of the structure can be accumulated linearly.

(2) When the fatigue damage accumulates to a certain value, the fatigue damage of structure occurs.

According to the theory of linear fatigue damage accumulation, $\operatorname{Dam}_{i}$ is defined as the damage rate of structure under the $\mathrm{i}^{\text {th }}$ level of stress. The total damage rate Dam is obtained by linearly superimposing the damage rates at different stress levels, as shown in the following equation (22). When Dam reaches 1.0, the structure is considered to be destroyed.

$$
\text { Dam }=\sum_{i} \operatorname{Dam}_{i}=\sum_{i} \frac{n\left(\Delta \sigma_{i}\right)}{N\left(\Delta \sigma_{i}\right)}
$$

Where, $n\left(\Delta \sigma_{i}\right), N\left(\Delta \sigma_{i}\right)$ are the number of cycles of alternating stress and the number of alternating stress cycles required for conductor breakage respectively when the range of stress is $\Delta \sigma_{i}$.

The Estimation of Aeolian Vibration Fatigue Life of Conductor. The maximum dynamic bending strain of the conductor in the locked frequency $f_{i}$ can be calculated by the energy balance method and the value of maximum bending stress can be obtained by the maximum dynamic bending strain. According to the formula (13), the number $N_{i}$ of alternating stress cycles required for conductor breakage can be produced, and $n_{i}$ can be calculated by the following formula.

$$
n_{i}=f_{i} t_{i}
$$

Where, $t_{i}$ is the total time of the conductor vibrating at frequency $f_{i}$ throughout the year. Thus, when the conductor vibrates at the frequency $f_{i}$, we can calculate the annual damage rate $\operatorname{Dam}_{i}$ as follows.

$$
\operatorname{Dam}_{i}=3.1536 \times 10^{7} \times\left\{\exp \left[-\left(f_{i}^{\text {low }} D / S_{t} c_{w}\right)^{k_{w}}\right]-\exp \left[-\left(f_{i}^{u p} D / S_{t} c_{w}\right)^{k_{w}}\right]\right\} f_{i} / N_{i}
$$

The vibration fatigue life of the conductor under the load spectrum condition is:

$$
\text { Life }=\psi \times 1 / \sum_{i} \operatorname{Dam}_{i}
$$

Where, $\psi$ is a correction coefficient which is used to correct the error caused by the average wind speed distribution function. Based on the above formulas, the relationship among the dynamic bending strain, the vibration frequency and the fatigue life can be established.

\section{Example Analysis}

Taking the anti-vibration design of a $1000 \mathrm{kV}$ UHV transmission line as an example, the main physical parameters of the conductor are shown in Table 1. 
Table 1. Parameters of the conductor.

\begin{tabular}{cc}
\hline Parameters & Value \\
\hline Conductor type & JLB20A-35 \\
Diameter $(\mathrm{mm})$ & 17.0 \\
Elastic Modulus $\left(\mathrm{N} / \mathrm{m}^{2}\right)$ & $14.72 \times 10^{10}$ \\
Sectional area $\left(\mathrm{mm}^{2}\right)$ & 182.8 \\
Mass per unit length $(\mathrm{kg} / \mathrm{m})$ & 1.2215 \\
Breaking force $(\mathrm{N})$ & 215520 \\
\hline
\end{tabular}

According to the above parameters, set the span as $500 \mathrm{~m}$ and the running tension of the conductor as $23 \%$ of the breaking force. Using the energy balance method to calculate the dynamic bending stress at the conductor clamp of the conductor without dampers, the curve of the dynamic bending stress varying with the frequency are shown in Fig. 4.

Setting the average annual wind speed as $3.0 \mathrm{~m} / \mathrm{s}$, the fatigue life of the conductor without dampers is 24.62 years and the fatigue life calculated by the nominal stress method is 19.84 years. In order to improve the service life of the transmission lines, the commonly used method is to install the dampers. In the following example, two FR4-type Stockbridge-type dampers are laid on both ends of the conductor.

The FR4-type Stockbridge-type damper's power characteristic curve is shown in Fig. 5.

After the addition of two FR4-type Stockbridge-type dampers, the dynamic bending stress at the conductor clamp varying with the frequency are shown in Fig. 6

After the installation of FR4-type Stockbridge-type dampers, the fatigue life of the conductor calculated by the fracture mechanics method is 85.34 years. The fatigue life calculated by the nominal stress method is 73.23 years. It can be seen that the aeolian vibration fatigue life will be significantly improved after the installation of FR4-type Stockbridge-type dampers.

Comparing the results calculated by the above two methods, it can be seen that the results obtained by the nominal stress method are relatively small, which is conservative. The fracture mechanics method considers the extension of the fretting fatigue crack and the result is more accurate.

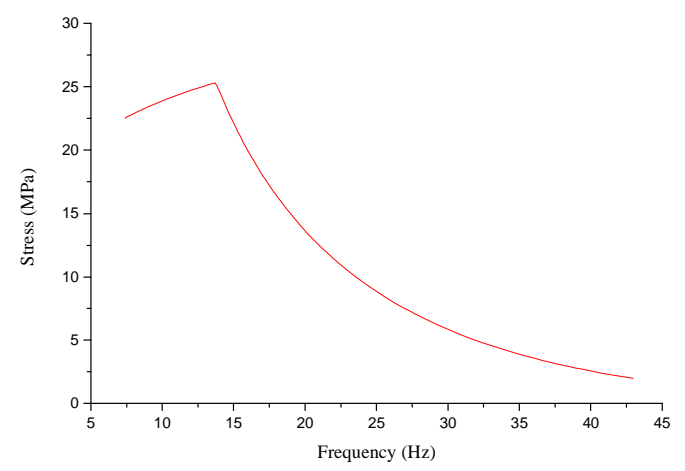

Fig. 4 Dynamic Bending Stress of The Conductor Without Dampers.

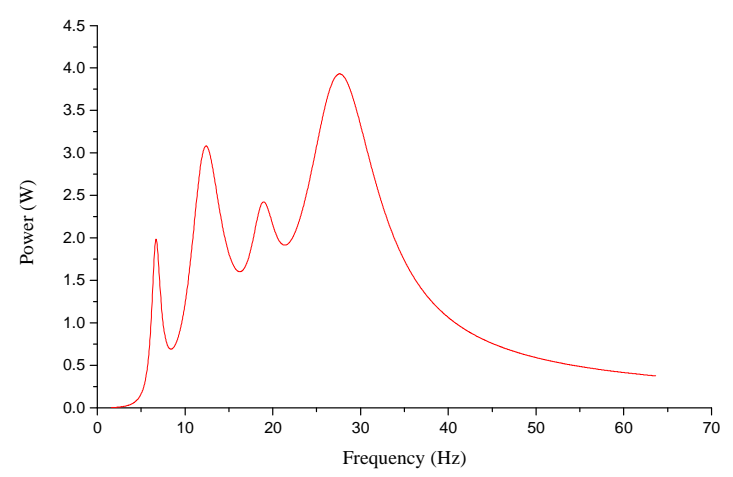

Fig. 5 Power Characteristic Curve of The FR4 Damper.

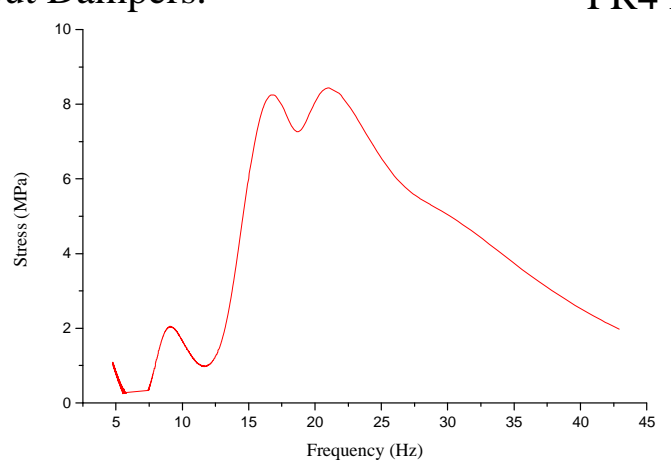

Fig. 6 Dynamic Bending Stress of The Conductor With FR4 Dampers. 


\section{Conclusion}

1. By applying the fracture mechanics method to the analysis and calculation of the fatigue life of the conductor and taking the fretting fatigue crack propagation of conductor into consideration, the results are more realistic.

2. Compared with the results obtained by the nominal stress method, the nominal stress method is more conservative. The aeolian vibration fatigue life of the conductor is improved after installing the Stockbridge-type dampers.

3. Based on the above research, the fracture mechanics method is introduced into the calculation and analysis of the fretting fatigue life of the conductor, which lays the foundation for the study of the anti-vibration scheme of the aeolian conductor vibration.

\section{References}

[1] T. X. Shao, Wires mechanics of transmission line, Beijing, 2003.

[2] Y. Q. Zheng, Aeolian vibration of transmission line, Beijing: Water conservancy and Hydropower press, 1987.

[3] S. P. Wang, D. Chen, Analysis of transmission lines vibration under well-distributed breeze loads, J. Wu Han Automot. Polytechnic Univ. 19(3) (1997) 70-74. (in Chinese)

[4] M. Ervik, A. Berg, A. Boelle, et al. Report on aeolian vibration of power overhead line, Electra, 12(4) (1989)41-77.

[5] J.T. Schmidt, G. Biedenhach, H. J. Krispin, Laboratory meas- urement of the power dissipation characteristics of aeolian vibration dampers,IEEE Trans. Power Deliv. 12(4) (1997) 1614-1621.

[6] M. L. Lu, J. K. Chan, An efficient algorithm for aeolian vibration of single conductor with multiple dampers, IEEE Trans. Power Deliv. 22(3) (2007) 1822-1829.

[7] Z. N. XU, Measure and life predicted of aeolian vibration on transmission lines, Electr. Power Construct. 12(10) (1991) 6-9. (in Chinese)

[8] D. Y. Kong, Research of transmission line aeolian vibration based on dynamic method.[PHD] Hua Zhong University of Science and Technology, 2009. (in Chinese)

[9] G. H. ZHU, Aeolian vibration and fatigue life of transimission lines, Harbin Institute of Technology, 2011. (in Chinese)

[10]H. B. CHEN, The fretting damage of high-voltage electrical power transmission conductor and its fretting fatigue life prediction, Hua Zhong University of Science and Technology, 2008. (in Chinese)

[11]G. Diana, A. Cigada, M. Belloli, Stockbridge-type damper effectiveness evaluation: Part IComparison between tests on span and on the shaker, IEEE Trans. Power Deliv. 18(4) (2003) 1462-1469.

[12]G. Diana, A. Manenti, C. Pirotta, Stockbridge-type damper effectiveness evaluation: Part IIThe influence of the impedance matrix terms on the energy dissipated, IEEE Trans. Power Deliv. 18(4) (2003) 1470-1477.

[13]M. L. LU, Computer simulation of dampers' power character, North. Electric Power Technol. 10(2) (1994) 1-4. (in Chinese) 